\title{
Post procedure analgesia with combined serratus anterior and parasternal plane blocks for subcutaneous implantable cardioverter-defibrillator (S-ICD) implantation - a case report. \\ St George's University Hospitals W/HS
}

\author{
E. Avagliano, L. Phylactides, A.I. Elbatran, A. Krol
}

DEPARTMENT OF ANAESTHESIA

\section{BACKGROUND AND AIMS}

- S-ICD implantation is associated with considerable intra and post-operative discomfort

- It involves: (i) incisions along the left sternal edge and left lateral chest wall (Fig. A) (ii) the creation of a subcutaneous pocket for the pulse generator (usually at the level of the $5^{\text {th }}$ and $6^{\text {th }}$ intercostal spaces in the mid-axillary line) (iii) tunnelling of the electrode from the medial to the lateral incisions and cephalad along the left sternal edge (Fig. B and C) (vi) ventricular fibrillation (VF) induction and defibrillation testing

- Implantation is usually performed under general anaesthesia (GA) and often requires opioid analgesia

- We present a case where GA was combined with ultrasound guided serratus anterior (US-SAPB) and parasternal plane blocks, for anterolateral chest wall and sternal edge analgesia respectively, as a means of reducing post-operative pain after S-ICD insertion

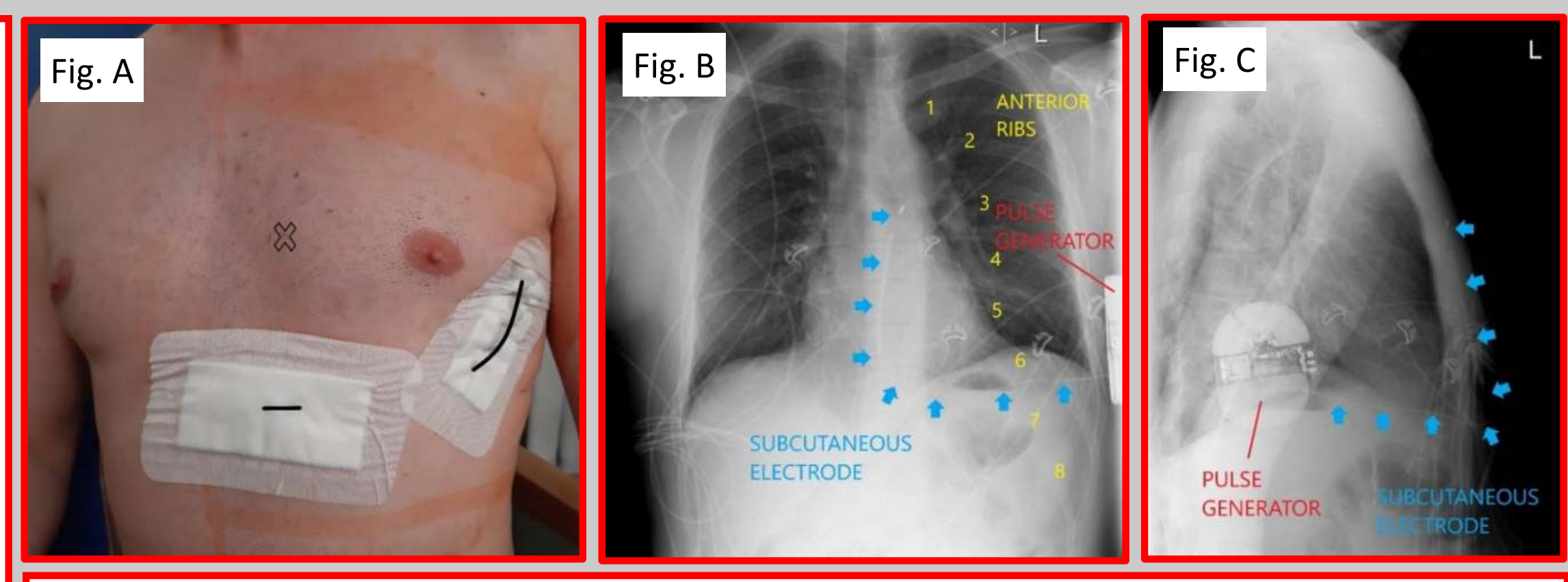

Fig. A: photograph of the patient post procedure. The black lines represent the incisions made. $\mathbf{X}$ marks the injection site of parasternal block. Fig. B \& C: Anterior-posterior and lateral chest X-rays taken the day after the procedure, showing the positions of the electrodes and pulse generator. In Fig. B, the ribs have been counted anteriorly.

Patient consent was obtained for photography and publication of the case report
- The patient was a 45 year old man undergoing elective S-ICD implantation for Brugada syndrome

- GA was induced with fentanyl $100 \mathrm{mcg}$ and propofol The local anaesthetic (LA) injections were performed in the supine position and with the left arm abducted

- A $80 \mathrm{~mm}$ SonoPlex (PAJUNK ${ }^{\circledR}$ ) needle was used with an in-plane approach. The LA solution was a 50:50 mixture of levobupivacaine $0.25 \%$ and lidocaine $1 \%$ with 1:200K adrenaline

The US-SAPB was performed first (Fig. D to F). $30 \mathrm{ml}$ of LA was injected superficial to the serratus anterior at the level of the $5^{\text {th }}$ intercostal space

- The parasternal block was subsequently performed (Fig. G to I). With the US probe in the parasagital plane $2 \mathrm{~cm}$ lateral to the sternal border, $20 \mathrm{ml}$ of LA was injected between the $4^{\text {th }}$ and $5^{\text {th }}$ ribs (Fig. A) in the subpectoral plane

Procedure duration was 1 hour and 45 minutes and was uneventful

Additional analgesia given intraoperatively included morphine $4 \mathrm{mg}$ and paracetamol $1 \mathrm{~g}$ (as per the anaesthetist's usual practice). A further $10 \mathrm{ml}$ of subcut levobupivacaine $0.25 \%$ was administered by the cardiologist at the end of the procedure

Data collected post-operatively included regular pain assessments and analgesia requirements

\section{METHODS}
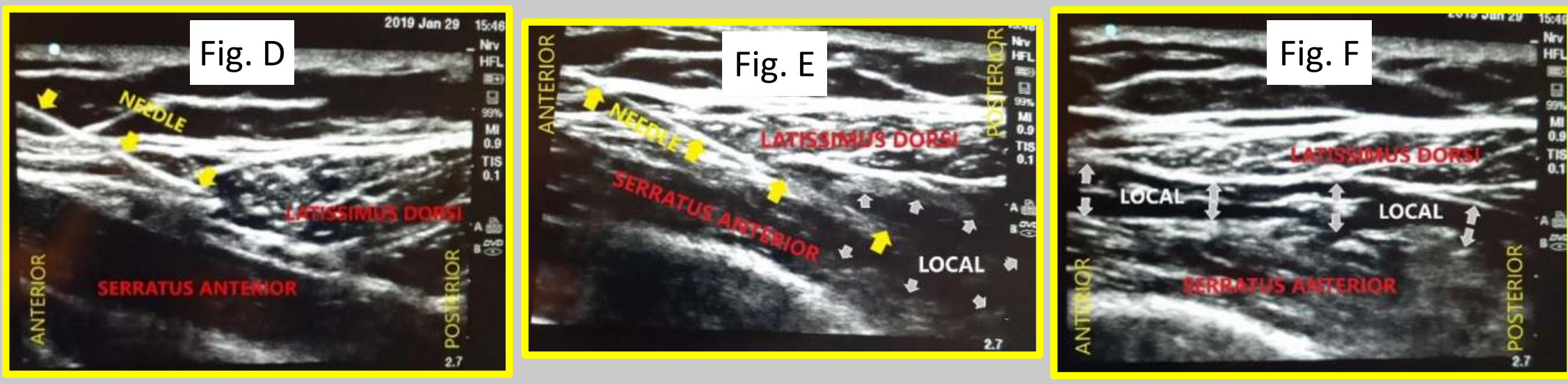

Ultrasound guided serratus anterior plane block - ultrasound images of procedure.

Fig. D: In plane approach of needle aiming for plane between latissimus dorsi (LD) and serratus anterior (SA) muscles. Fig. E: Injection and spread of local anaesthetic (LA) - some intramuscular injection into SA noted. Fig. F: Post injection US scan after needle removal showing LA occupying plane between SA and LD and appearing to spread posteriorly

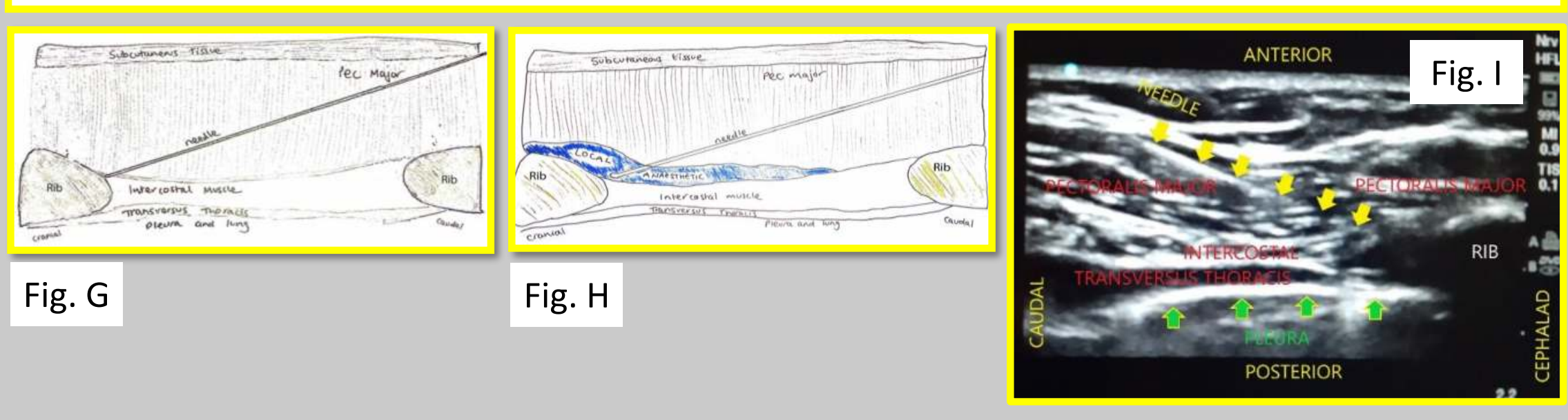

Ultrasound guided parasternal plane block - schema and ultrasound image of procedure Fig. G \& H: Schematic representation of anatomy, needle positioning and injection / spread of LA. Fig. I: Ultrasound image of actual procedure showing needle positioning prior to LA injection

\section{RESULTS}

The patient required no additional analgesia in the Post Anaesthesia Care Unit (PACU) and remained pain free overnight

He did not experience any discomfort until 6am the following morning, when he complained of moderate pain localised at the site of the xiphoid incision. There was no pain in the region of the lateral incision and subpectoral pocket

Oral analgesia (codeine, ibuprofen, paracetamol) was sufficient to treat the discomfort

\section{CONCLUSIONS}

- The combination of serratus anterior and parasternal plane blocks provides adequate post-operative analgesia for the creation of the pocket and for tunnelling the electrode. Additional analgesic strategies may be needed to cover the xiphoid incision

For the procedure to be performed under regional anaesthesia, avoiding GA, additional considerations might include the timing of the block, techniques to prolong block duration and the need for sedation during VF induction and defibrillator testing

\section{REFERENCES}

1. Successful cases of S-ICD implantation performed under the serratus plane block. Ueshima H. et al. Journal of Clinical Anaesthesia (2016) 33: 147-148

2. Feasibility of subcutaneous implantable cardioverter-defibrillator implantation with opioid sparing truncal plane blocks and deep sedation. Miller M.A. et al. J Cardiovasc Electrophysiol (2019) 30:141-148

3. Anaesthesia for subcutaneous implantable cardioverter-defibrillator implantation: Perspectives from the clinical experience of a U.S. panel of physicians. Essandoh M.K. et al. Pacing Clin Electrophysiol (2018) 41: 807-816 